\title{
Finger and Palm Dynamic Pressure Monitoring for Basketball Shooting
}

\author{
Chiao-Fang Hung, ${ }^{1}$ Chung-Chiang Chen, ${ }^{2}$ Shin-Hung Lin, ${ }^{1}$ and Tien-Kan Chung ${ }^{1,3}$ \\ ${ }^{1}$ Department of Mechanical Engineering, National Chiao Tung University, Hsinchu 30010, Taiwan \\ ${ }^{2}$ Office of Physical Education, National Chiao Tung University, Hsinchu 30010, Taiwan \\ ${ }^{3}$ International College of Semiconductor Technology, National Chiao Tung University, Hsinchu 30010, Taiwan
}

Correspondence should be addressed to Tien-Kan Chung; tkchung@nctu.edu.tw

Received 3 February 2017; Accepted 4 May 2017; Published 23 May 2017

Academic Editor: Andrea Cusano

Copyright (C) 2017 Chiao-Fang Hung et al. This is an open access article distributed under the Creative Commons Attribution License, which permits unrestricted use, distribution, and reproduction in any medium, provided the original work is properly cited.

\begin{abstract}
This study verified general inferences on the finger and palm pressure distribution of a basketball player in the moment before that player shoots a basketball through a scientific qualitative testing method. We mounted the sensor on the hands of college basketball players and monitored the dynamic pressure of each player's hand while the player threw a basketball. The dynamic pressure distribution of the fingers and palm of a basketball player throwing a ball can be verified. According to the experimental results, college basketball players typically use the index finger to control the direction and power of force in the moment before shooting a basketball. This study successfully used a scientific qualitative test method to monitor the dynamic pressure of the fingers and palms of basketball players and verified the general inference that a typical basketball player mainly uses the index finger to control the direction and power of force in the moment before throwing a ball. In the future, this study, measuring the dynamic pressure distribution of the fingers and palm, can be applied to simulate hand manipulation in many biomedical and robotic applications.
\end{abstract}

\section{Introduction}

A variety of pressure sensors are currently used in the industry, mainly piezoresistive, capacitive, and piezoelectric sensors. Among these, piezoresistive pressure sensors [1-5] have scope for use in practical applications for sensing the human body [6-12], such as all kinds of haptic sensing of the human body [13-17] including haptic sensing of fingers and palms [18-20].

Recently, a general inference on the finger and palm pressure distribution of a basketball player in the moment before shooting a basketball has been derived: a typical basketball player uses the index finger to control the direction and power of force in the moment before throwing a basketball. To support this general inference, some researchers claimed that, according to the principles of human-motion mechanics, the basketball player uses the index finger to control the direction and the power of the shooting force in the moment right before shooting a basketball [21]. This is because the index finger is the longest finger and the working distance is the longest distance, and both are helpful to control the direction and the power of the force for shooting the basketball. However, currently, no related experimental dynamic pressure sensing/monitoring research has been completely conducted on a basketball player using the index finger in the moment before throwing a basketball. In other words, the aforementioned general inference has not been scientifically verified. To qualitatively measure and scientifically verify this general inference, we used the TekScan Grip system [22] as a pressure sensor to monitor the dynamic pressure of the fingers and palms of basketball players while they were throwing balls. By analyzing each player's result from the dynamic pressure monitoring of the fingers and palm, we verified the aforementioned general inference by using a scientific qualitative test method. Furthermore, our verified approach measuring the dynamic pressure distribution of 


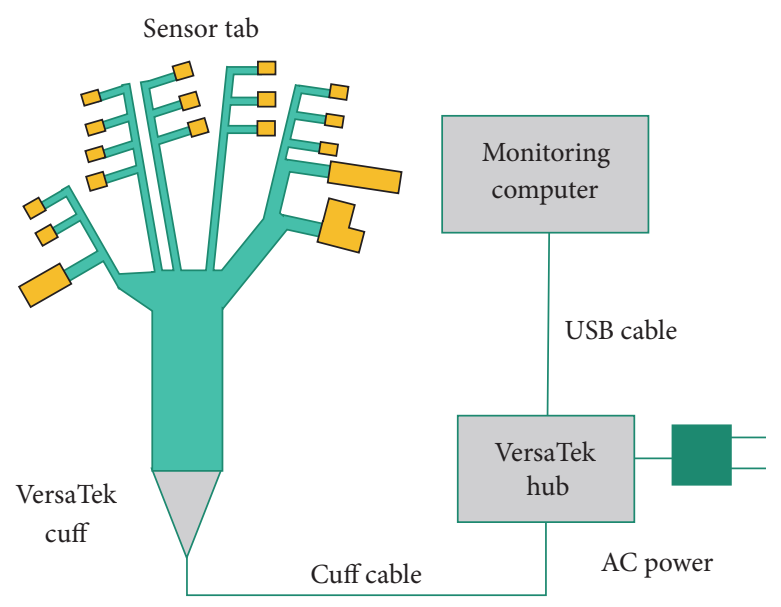

(a)

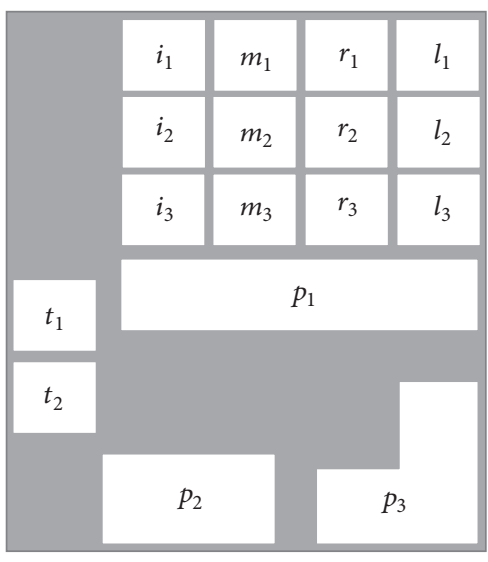

(b)

FIGURE 1: (a) Illustration of TekScan Grip system configuration. (b) Illustration of the finger and palm pressure distribution.

the fingers and palm can be applied in many biomedical and robotic applications for simulating hand manipulation. For example, our approach can be modified to design an anthropomorphic robot hand and measure the pressure distributions of the robot hand [23], to achieve haptic feedback (tactile and force) $[24,25]$ in robotic surgery using the da Vinci robotic system [26], and to measure the hand-arm biodynamic response for real power tool handles [27]. Due to these, our approach not only can be used for current sport-science applications, but also can provide an important design reference for developing future hand-manipulation stimulating technologies in medical and robotic applications. The design, testing, results, and discussion of our approach are described in the following sections.

\section{Design}

The TekScan Grip system is illustrated in Figure 1(a). The main devices constituting this system are the sensor tab, VersaTek cuff, VersaTek hub, and a monitoring computer. The area of the sensor tab comprises 18 independent pressure sensing areas, which can monitor the pressure of the fingers and palm. The connection configuration of the TekScan Grip system is as follows. First, the sensor tab is connected to the VersaTek cuff. The VersaTek cuff can transfer the signals from the sensor tab to the cuff cable. Subsequently, the cuff cable is connected to the VersaTek hub. Finally, the AC power of the VersaTek hub is connected to the TekScan Grip system, and the VersaTek hub is connected to the monitoring computer with a USB cable to monitor the pressure distribution of the fingers and palm. The finger and palm pressure distribution is shown in Figure 1(b); the figure shows the pressure distribution of each area of the palm $\left(p_{1}\right.$, $p_{2}$, and $\left.p_{3}\right)$ and each segment of the thumb $\left(t_{1}\right.$ and $\left.t_{2}\right)$, index finger $\left(i_{1}, i_{2}\right.$, and $\left.i_{3}\right)$, middle finger $\left(m_{1}, m_{2}\right.$, and $\left.m_{3}\right)$, ring finger $\left(r_{1}, r_{2}\right.$, and $\left.r_{3}\right)$, and little finger $\left(l_{1}, l_{2}\right.$, and $\left.l_{3}\right)$. By using the TekScan Grip system, we can monitor and record the pressure of the fingers and palm of a basketball player while that player is throwing a ball and determine the pressure of each area of the palm and each segment of each finger.

\section{Testing}

To measure the actual behavior of basketball players, we asked the college basketball players to wear the sensor system and perform ball-shooting, and we monitored and recorded the pressure levels of their right hands. To facilitate the sensor installation on the hands of the basketball players, adjust the sensor positions, and avoid hygienic issues, we mounted the sensor tab on their gloves (latex gloves, thickness $0.15 \mathrm{~mm}$; Taihang Enterprise Co. Ltd., Hsinchu, Taiwan), following the procedure of Mastalerz et al. [6]. Furthermore, to prevent the VersaTek cuff from affecting the operation of the basketball player while shooting the ball, we carefully affixed the VersaTek cuff to the upper arm of each player. After completing these configurations of the TekScan Grip system, we investigated the finger and palm pressure distributions of the players by analyzing the recording of the sensor system. In addition, the players who we chose for test have common features: college student, about 20 years old, having a little nonprofessional basketball training (e.g., school team member or department team member), and about $175-180 \mathrm{~cm}$ height with a similar stature. Due to the choosing criteria, the test results are comparable.

\section{Results and Discussion}

The records of the finger and palm dynamic pressure distributions of college basketball players, that is, the school basketball team member and the department basketball team member, are shown in Figures 2 and 3, respectively. We can analyze the results of the dynamic pressure monitoring of the fingers and palm in three stages (first, holding the ball; second, lifting the ball overhead; and third, the moment 


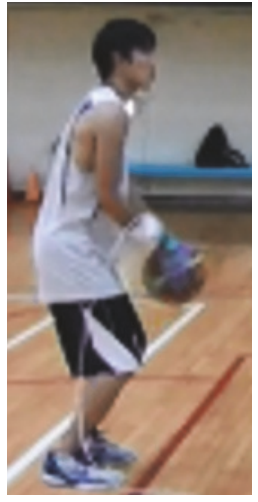

(a)

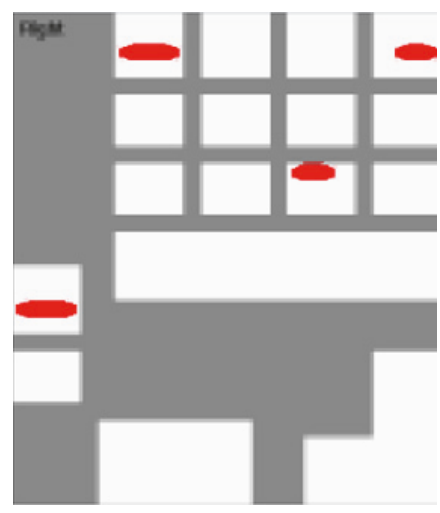

(b)

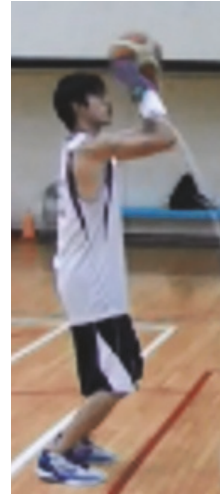

(c)

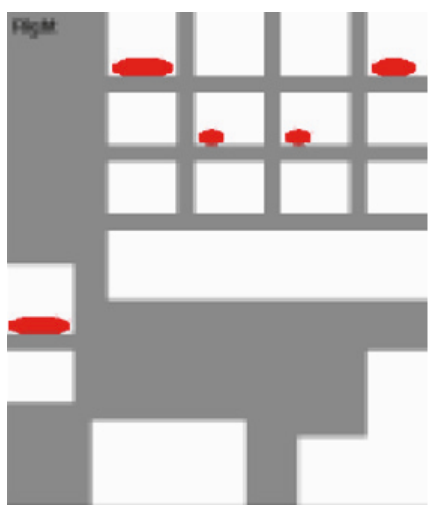

(d)

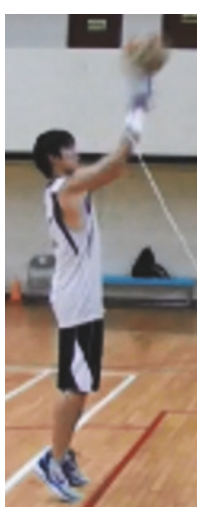

(e)

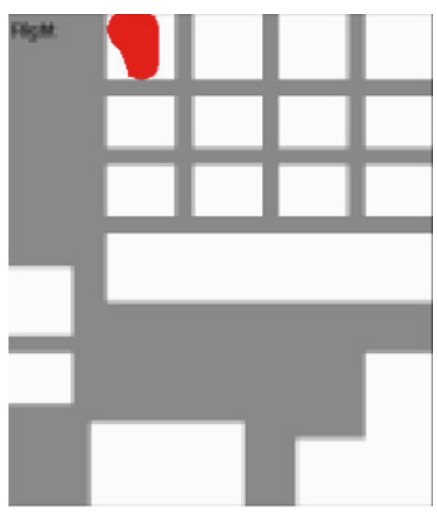

(f)

FIGURE 2: Photographs while shooting a ball and the finger and palm pressure distribution of the school basketball team member at the following stages: $(a, b)$ holding the ball, $(c, d)$ lifting the ball overhead, and $(e, f)$ the moment before shooting the ball.

before shooting the ball). By comparing these distributions of dynamic pressure of the finger and palm, we obtained the following results. While holding the ball, most of the fingers of both basketball players had a high-pressure distribution, as shown in Figures 2(a), 2(b), 3(a), and 3(b). Because each player's habits are different, the pressure distributions were also different. While lifting the ball overhead, the finger and palm pressure distribution of both basketball players was observed on all fingers, as shown in Figures 2(c), 2(d), $3(\mathrm{c})$, and $3(\mathrm{~d})$. Finally, in the moment before shooting the ball, both basketball players mainly used the index finger to control the direction and power for shooting the ball, as shown in Figures 2(e), 2(f), 3(e), and 3(f). These results are consistent with the aforementioned general inference. Thus, we successfully verified the general inference on the finger and palm pressure distribution by using the scientific qualitative test method.

\section{Conclusions}

By using the piezoresistive pressure sensing/monitoring system, we successfully verified the general inference on the finger and palm dynamic pressure distribution in the moment while the basketball players throw the ball (i.e., during ball-shooting). The experimental results of the dynamic pressure monitoring revealed that the college basketball players mainly used the index finger to control the direction and power for shooting the ball. These results not only verified the general inference but also could provide a reference for current basketball sport science. Moreover, our approach can provide an important design reference for future handmanipulation simulating technologies in medical and robotic applications.

\section{Conflicts of Interest}

The authors declare that there are no conflicts of interest regarding the publication of this paper.

\section{Authors' Contributions}

Chiao-Fang Hung and Chung-Chiang Chen contributed equally to this work.

\section{Acknowledgments}

This work is supported by the Ministry of Science and Technology, Taiwan (Grant no. 105-2628-E-009-001-MY2). 


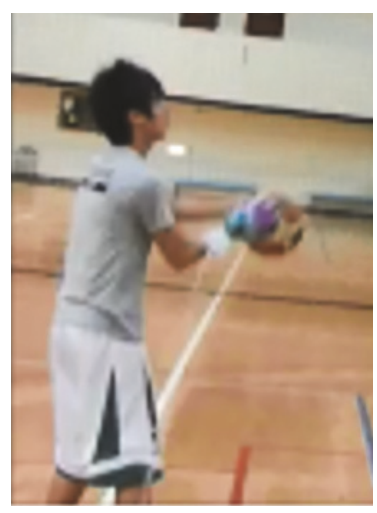

(a)

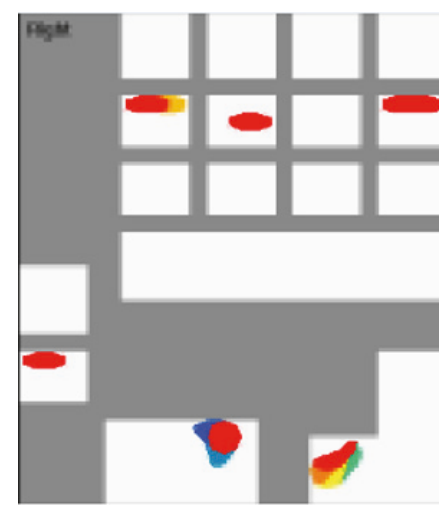

(b)

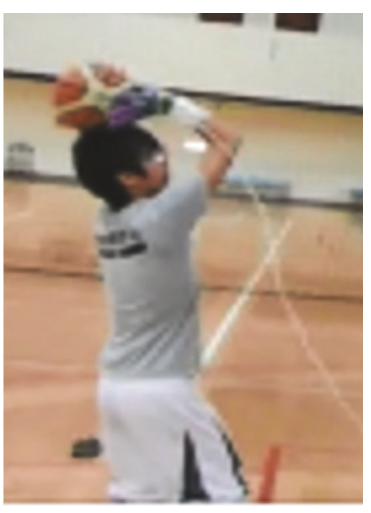

(c)

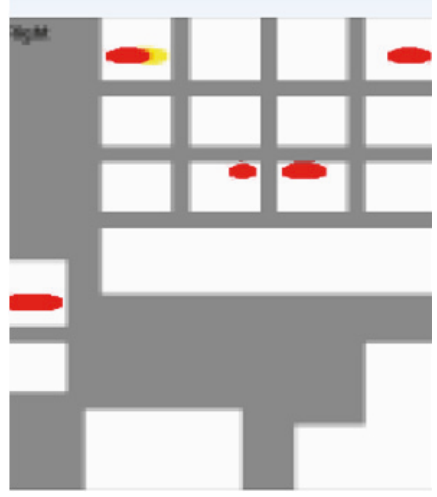

(d)

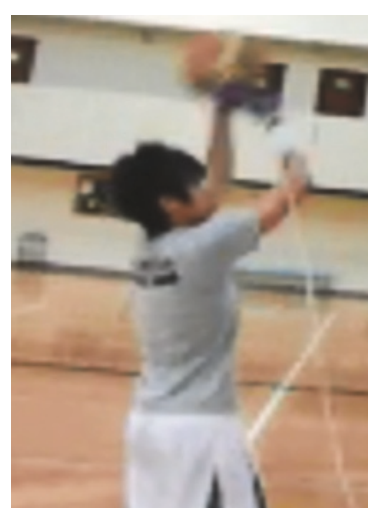

(e)

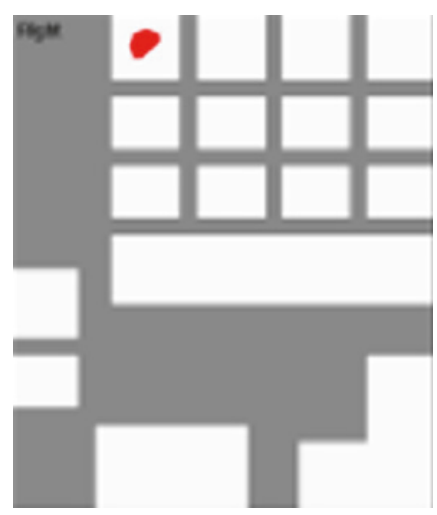

(f)

FIGURE 3: Photographs while shooting a ball and the finger and palm pressure distribution of the department basketball team member at the following stages: $(a, b)$ holding the ball, $(c, d)$ lifting the ball overhead, and $(e, f)$ the moment before shooting the ball.

\section{References}

[1] J. Zavickis, M. Knite, K. Ozols, and G. Malefan, "Development of percolative electroconductive structure in piezoresistive polyisoprene-nanostructured carbon composite during vulcanization," Materials Science \& Engineering C-Materials for Biological Applications, vol. 31, no. 2, pp. 472-476, 2011.

[2] H.-B. Yao, J. Ge, C.-F. Wang et al., "A flexible and highly pressure-sensitive graphene-polyurethane sponge based on fractured microstructure design," Advanced Materials, vol. 25, no. 46, pp. 6692-6698, 2013.

[3] E. Laukhina, R. Pfattner, L. R. Ferreras et al., "Ultrasensitive piezoresistive all-organic flexible thin films," Advanced Materials, vol. 22, no. 9, pp. 977-981, 2010.

[4] S. Stassi, V. Cauda, G. Canavese, and C. F. Pirri, "Flexible tactile sensing based on piezoresistive composites: a review," Sensors, vol. 14, no. 3, pp. 5296-5332, 2014.

[5] J. Sosa, J. A. Montiel-Nelson, R. Pulido, and J. C. GarciaMontesdeoca, "Design and Optimization of a Low Power Pressure Sensor for Wireless Biomedical Applications," Journal of Sensors, vol. 2015, Article ID 352036, 2015.

[6] A. Mastalerz, E. Nowak, I. Palczewska, and E. Kalka, "Maximal grip force during holding a cylindrical handle with different diameters," Human Movement, vol. 10, no. 1, pp. 26-30, 2009.

[7] S. Chun, H. Jung, Y. Choi, G. Bae, J. P. Kil, and W. Park, "A tactile sensor using a graphene film formed by the reduced graphene oxide flakes and its detection of surface morphology," Carbon, vol. 94, pp. 982-987, 2015.

[8] A. J. Shaw, B. A. Davis, M. J. Collins, and L. G. Carney, "A Technique to Measure Eyelid Pressure Using Piezoresistive Sensors," IEEE Transactions on Biomedical Engineering, vol. 56, no. 10, pp. 2512-2517, 2009.

[9] J. C. Roberts, A. C. Merkle, P. J. Biermann et al., "Computational and experimental models of the human torso for nonpenetrating ballistic impact," Journal of Biomechanics, vol. 40, no. 1, pp. 125-136, 2007.

[10] Y. Wang, L. Wang, T. Yang et al., "Wearable and highly sensitive graphene strain sensors for human motion monitoring," Advanced Functional Materials, vol. 24, no. 29, pp. 4666-4670, 2014.

[11] M. Melnykowycz, M. Tschudin, and F. Clemens, "Piezoresistive soft condensed matter sensor for body-mounted vital function applications," Sensors (Switzerland), vol.16, no. 3, article no. 326, 2016.

[12] D. Giovanelli and E. Farella, "Force sensing resistor and evaluation of technology for wearable body pressure sensing," Journal of Sensors, vol. 2016, Article ID 9391850, 2016.

[13] A. Drimus, G. Kootstra, A. Bilberg, and D. Kragic, "Design of a flexible tactile sensor for classification of rigid and deformable objects," Robotics and Autonomous Systems, vol. 62, no. 1, pp. 315, 2014.

[14] R. E. Fan, M. O. Culjat, C.-H. King et al., "A haptic feedback system for lower-limb prostheses," IEEE Transactions on Neural 
Systems and Rehabilitation Engineering, vol. 16, no. 3, pp. 270277, 2008.

[15] M. Y. Chuah and S. Kim, "Enabling force sensing during ground locomotion: A bio-inspired, multi-axis, composite force sensor using discrete pressure mapping," IEEE Sensors Journal, vol. 14, no. 5, pp. 1693-1703, 2014.

[16] J. Castellanos-Ramos, R. Navas-González, H. Macicior, T. Sikora, E. Ochoteco, and F. Vidal-Verdú, "Tactile sensors based on conductive polymers," Microsystem Technologies, vol. 16, no. 5, pp. 765-776, 2010.

[17] C.-H. King, M. O. Culjat, M. L. Franco et al., "Tactile feedback induces reduced grasping force in robot-assisted surgery," IEEE Transactions on Haptics, vol. 2, no. 2, pp. 103-110, 2009.

[18] J. Hwang, J. Jang, K. Hong et al., "Poly(3-hexylthiophene) wrapped carbon nanotube/poly(dimethylsiloxane) composites for use in finger-sensing piezoresistive pressure sensors," Carbon, vol. 49, no. 1, pp. 106-110, 2011.

[19] C. Antfolk, C. Balkenius, G. Lundborg, B. Rosen, and F. Sebelius, "A tactile display system for hand prostheses to discriminate pressure and individual finger localization," Journal of Medical and Biological Engineering, vol. 30, no. 6, pp. 355-359, 2010.

[20] A. M. Almassri, W. Z. W. Hasan, and S. A. Ahmad, "Pressure sensor: state of the art, design, and application for robotic hand," Journal of Sensors, vol. 2015, Article ID 846487, 12 pages, 2015.

[21] L. Zhen, L. Wang, and Z. Hao, "A biomechanical analysis of basketball shooting," International Journal of Simulation-Systems, Science \& Technology, vol. 16, no. 3B, p. 1, 2015.

[22] TekScan Grip System. Available online: https://www.tekscan .com/products-solutions/systems/grip-system.

[23] H. Kawasaki, T. Komatsu, and K. Uchiyama, "Dexterous anthropomorphic robot hand with distributed tactile sensor: Gifu hand II," IEEE/ASME Transactions on Mechatronics, vol. 7, no. 3, pp. 296-303, 2002.

[24] C. Diaz and S. Payandeh, "Multimodal Sensing Interface for Haptic Interaction," Journal of Sensors, vol. 2017, Article ID 2072951, 24 pages, 2017.

[25] C. Wu, A. Song, Y. Ling, N. Wang, and L. Tian, "A control strategy with tactile perception feedback for emg prosthetic hand," Journal of Sensors, vol. 2015, Article ID 869175, 15 pages, 2015.

[26] M. Culjat, C.-H. King, M. Franco, J. Bisley, W. Grundfest, and E. Dutson, "Pneumatic balloon actuators for tactile feedback in robotic surgery," Industrial Robot, vol. 35, no. 5, pp. 449-455, 2008.

[27] M. Kalra, S. Rakheja, P. Marcotte, K. N. Dewangan, and S. Adewusi, "Feasibility analysis of low-cost flexible resistive sensors for measurements of driving point mechanical impedance of the hand-arm system," International Journal of Industrial Ergonomics, vol. 49, pp. 44-52, 2015. 


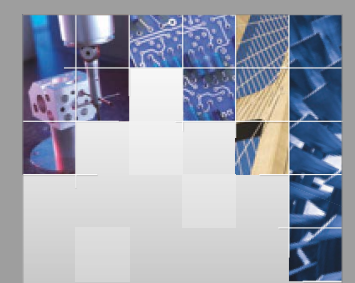

\section{Enfincering}
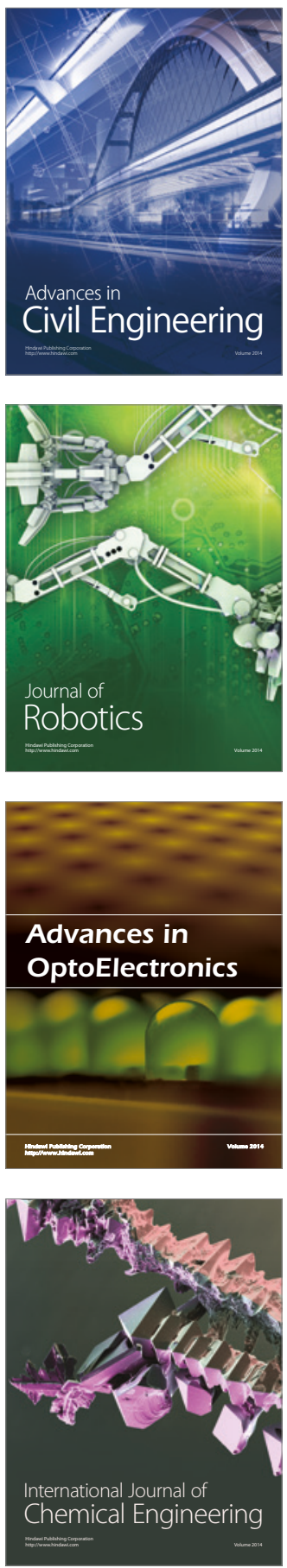

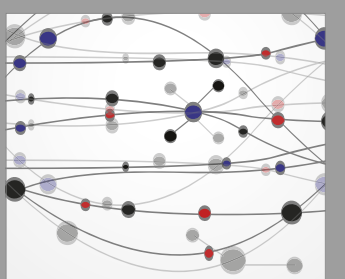

The Scientific World Journal

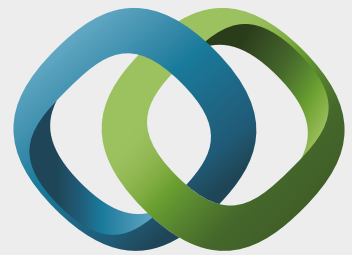

\section{Hindawi}

Submit your manuscripts at

https://www.hindawi.com
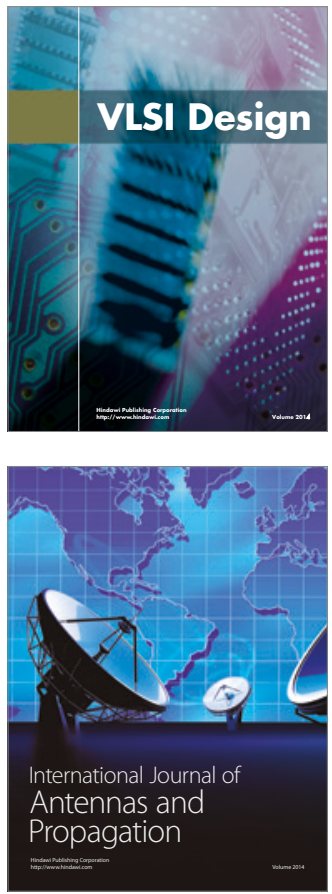

\section{Rotating}

Machinery
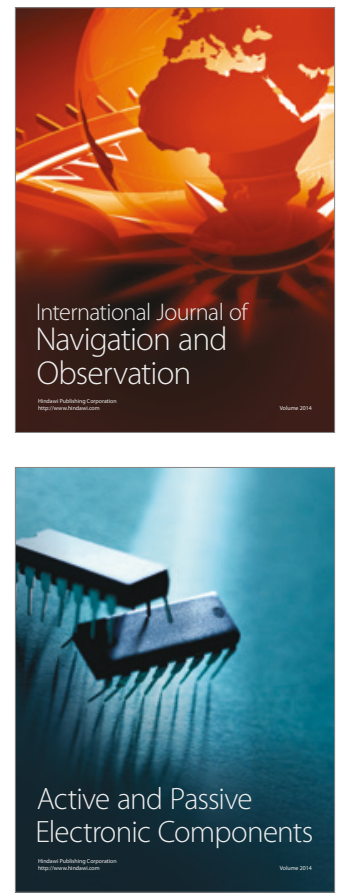
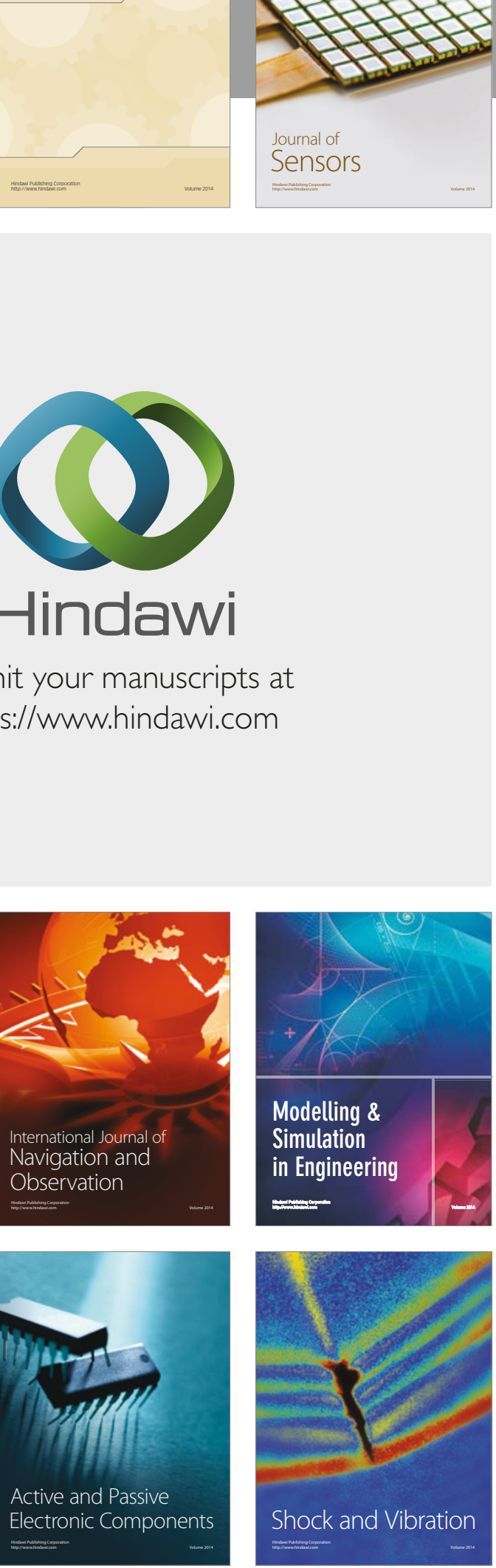
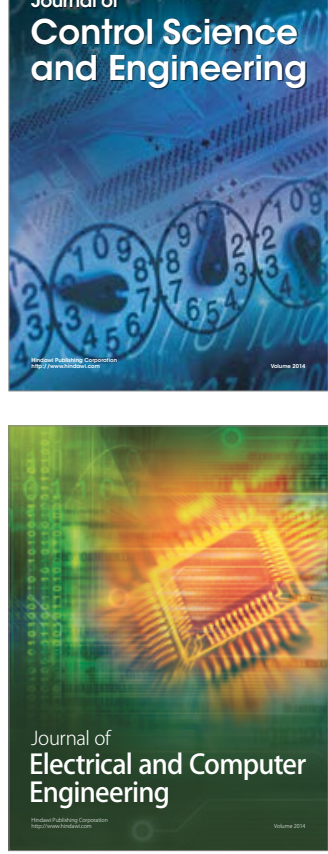

Distributed

Journal of

Control Science

and Engineering
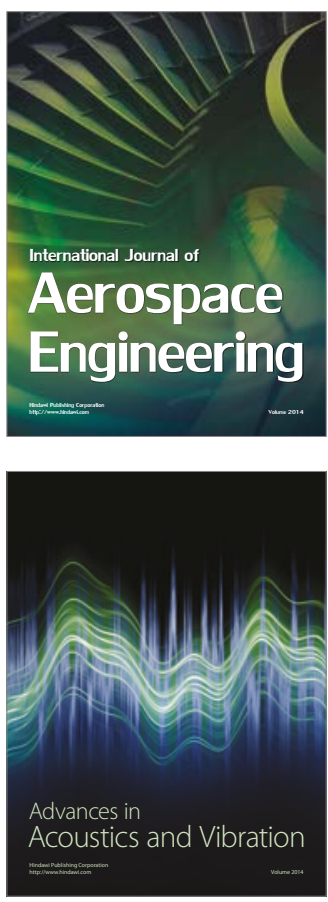

Sensor Networks 